\title{
Documentos
}

\section{DOLOR LUMBAR RELACIONADO AL EMBARAZO}

\author{
Milan Munjin L. ${ }^{1}$, Francisco llabaca G. ${ }^{1}$, Juan Rojas B. ${ }^{2}$ \\ ${ }^{1}$ Equipo de Columna Vertebral, Servicio de Traumatología, Hospital del Trabajador de Santiago. ${ }^{2 B e c a d o}$ de Ginecología \\ y Obstetricia, Universidad del Desarrollo, Hospital Padre Hurtado-Clínica Alemana.
}

\section{RESUMEN}

El dolor lumbar ha sido considerado la complicación más frecuente del embarazo, a pesar de ello, su etiopatogenia aún es controvertida. La prevalencia en nuestro medio es desconocida, y el tratamiento se basa para muchos médicos solo en el reposo, medida que no tiene sustento en la literatura. En esta revisión, nos centramos en la historia clínica, terminología, mecanismos fisiológicos teóricamente implicados, factores de riesgo, prevalencia y proponemos un enfoque de tratamiento del dolor lumbar asociado al embarazo.

\section{PALABRAS CLAVES: Dolor lumbar, embarazo}

\section{SUMMARY}

The low back pain has been considered the most frequent complication of pregnancy, despite this fact, etiology and pathophysiology is unclear at present time. The prevalence in our country is unknown, and the treatment is based on in rest-periods for the most physicians, instead literature rationality. In our research, based in the literature, we focus in the history, terminology, theoretical physiological mechanism, risk factors, prevalence and we propose pathways for treatment of pregnancy-related low back pain.

\section{KEY WORDS: Low back pain, pregnancy}

\section{INTRODUCCIÓN}

El dolor lumbar relacionado al embarazo, a pesar que es conocido desde hace muchos siglos, solo ha cobrado importancia en los últimos años por el impacto que tiene en la calidad de vida de la embarazada y en los costos económicos que involucra. En las últimas dos décadas hubo una explosión de artículos sobre el tema, junto a una gran cantidad de pacientes que consultaban por dolor lumbar, el que es considerado por la sociedad, como un evento patológico.

El conocimiento de esta complicación es una necesidad tanto para el gineco-obstetra, como para el traumatólogo, a quien es derivada para una segunda opinión o tratamiento. Los distintos artículos en la literatura internacional varían de gran forma al exponer la posible fisiopatología, la prevalencia, los factores de riesgo y también el tratamiento, por lo que una lectura parcializada de esta puede dar una visión equívoca.

El objetivo de esta comunicación es revisar la literatura en torno al dolor lumbar relacionado al embarazo, en sus aspectos etiológicos, fisiopatológicos y terapéuticos.

\section{HISTORIA}

Existe evidencia histórica que el dolor en la región lumbar y el anillo pélvico relacionado al 
embarazo eran problemas conocidos y descritos hace ya muchos siglos. Hipócrates (siglo IV A.C.) describe la teoría de la "disjunctio pelvica" que, según el padre de la medicina, consistía en el ensanchamiento de la sínfisis pubiana que solo ocurría en el primer parto y quedaba ensanchada para los siguientes embarazos, relacionando este hecho a la aparición de dolor. Esta teoría se vio reforzada por numerosos autores, entre ellos Vesalius, Severin Pinean, Ambroise Paré, Albinus de Leyden, William Hunter, Velpeau, Jacquemier, Baudelocque, Lenoir, Luschka y muchos otros. Sus visiones, eso si, eran marcadamente diferentes: unos creían que la relajación pélvica era normal y un fenómeno constante, mientras otros la consideraban excepcional y patológica (1).

Fue en el siglo XVII cuando el concepto de debilitamiento de las articulaciones sacroilíacas y sínfisis pubiana durante el embarazo tomó mayor relevancia. En ese momento se consideró a este proceso como necesario, siendo un importante prerrequisito para el ensanchamiento del canal del parto. El mecanismo por el cual el ensanchamiento era producido generaba grandes discusiones en la época. No fue hasta el siglo XIX, cuando se puso mayor atención en el dolor en la región lumbar y cintura pélvica durante y después del embarazo. En 1870, Snelling describe el "Síndrome Pélvico" de la siguiente forma: "la afección aparentemente consiste en la relajación de las articulaciones de la pelvis, iniciándose bruscamente luego del parto o en forma gradual durante el embarazo, permitiendo un grado de movilidad de los huesos pélvicos que entorpece la marcha y ocasiona las sensaciones de dolor más peculiares y alarmantes" (1).

Al inicio del siglo XX, la mayor parte de los autores aceptaban la visión que las articulaciones pélvicas se ablandaban y relajaban durante el embarazo, pero hasta ese momento no se habían realizado estudios sobre la frecuencia ni el grado de separación, así como tampoco se había investigado cuanto influía en la aparición del dolor. Brehm en 1928 intentó establecer grados de separación de la sínfisis púbica, dividiendo su población en dos grupos, el primero de entre 9 y $20 \mathrm{~mm}$ de separación con pocos síntomas y el segundo con $20 \mathrm{~mm}$ o más y marcada sintomatología. Abramson y cols (2), publican la pregunta en 1932 sobre si existe relación entre la relajación de la cintura pélvica y el dolor presente en el embarazo. Este hecho fue crucial, desde ese momento se consideró al dolor lumbar y de la cintura pélvica en el embarazo como un proceso patológico aisla- do. Para este efecto se le llamó "Insuficiencia Pélvica" y se describió (3) como un conjunto de síntomas objetivos y subjetivos, entre ellos: la fatiga sin causa clara, dolor con eventual irradiación, dificultad en la marcha o para dar pasos completos, dificultad para darse vuelta en la cama y para levantarse desde una silla. Solo en 1962, Walde (4) describe las diferencias entre el dolor pélvico y el lumbar durante el embarazo. El estudio del dolor lumbar y de la cintura pélvica relacionado al embarazo ha tenido un aumento muy importante las últimas décadas ya que se toma conocimiento del posible impacto de esta patología en la calidad de vida y los costos que tiene la enfermedad para la sociedad. Con el impacto económico de por medio se inician una serie de estudios, en especial en población escandinava, los cuales describiremos a continuación.

\section{TERMINOLOGÍA}

Existe una extensa lista de términos utilizados en la literatura para describir el dolor lumbar y pélvico relacionado con el embarazo (5), un problema importante es que muchos de ellos indican un mecanismo patológico sin aun estar esclarecida la real etiología de la enfermedad (Tabla I). Además gran cantidad de términos adicionan temporalidad al fenómeno, siendo esto innecesario y limitante en nuestra opinión, al igual que lo descrito en la literatura mas reciente (5), debiera utilizarse el término "relacionado", lo cual incluye el dolor que aparece durante el embarazo y también el posparto. Múltiples estudios han mostrado que existirían dos subgrupos dentro de la patología descrita, debido a la ubicación del dolor, diferencias en la incidencia y en la evolución del cuadro, por lo tanto, según la literatura debe diferenciarse entre el dolor en la cintura pélvica relacionado con el embarazo (pregnancy-related pelvis girdle pain or PPP) y el dolor lumbar relacionado con el embarazo (pregnancy-related low back pain or PLBP). Muchos estudios no realizan esta diferenciación por lo cual nos referiremos, en esos casos, como dolor lumbopélvico relacionado con el embarazo.

\section{FISIOPATOLOGÍA}

Aunque el dolor lumbar y pélvico es la complicación mas frecuente del embarazo, su etiología es desconocida y su fisiopatología pobremente entendida (6). Los cambios fisiológicos normales del embarazo afectarían también a la columna, 
Tabla I

TÉRMINOS USADOS DESDE 1900 PARA DESCRIBIR EL DOLOR LUMBAR Y PÉLVICO RELACIONADO AL EMBARAZO

\begin{tabular}{lll}
\hline Terminología propuesta & Autor & Año de publicación \\
\hline Relaxation of the pelvic joints in pregnancy & Abramson D & 1934 \\
Pelvic osteo-arthropathy & Young J & 1939 \\
Insufficientia pelvis gravidarum et puerperarum & Genell S & 1949 \\
Pelvic insufficiency & Berezin D & 1954 \\
Backache during pregnancy & Wickstrom J & 1955 \\
Pelvic girdle relaxation & Slate WG & 1960 \\
Pelvic arthropathy & Percy-Lancaster R & 1969 \\
Pelvic instability & Lindsel H & 1984 \\
Postpartum pelvic arthropathy & Driessen E & 1987 \\
Spinal and pelvic insufficiency & Berg G & 1988 \\
Symptom-giving pelvic girdle relaxation & Dietrichs E & 1991 \\
Pelvic pain and pelvic joint instability & Saugstad LF & 1991 \\
Posterior pelvic pain in pregnancy vs back pain in pregnancy & Ösgaard HC & 1994 \\
Pelvic pain in pregnancy vs low back pain in pregnancy & Endresen EH & 1995 \\
Peripartum pelvic pain & Mens JM & 1996 \\
Pregnancy-related back and pelvic pain & Björklund K & 1999 \\
Back pain postpartum & Nilsson-Wikmar L & 1999 \\
Pregnancy-related pelvic joint pain & Albert H & 2000 \\
Pregnancy-related low back pain & Mens JMA & 2000 \\
Pregnancy-related pelvic pain & Albert H & 2001 \\
Posterior pelvic pain since pregnancy & Mens JM & 2002 \\
Posterior pelvic pain after pregnancy & Mens JM & 2002 \\
Lumbar back and posterior pelvic pain during pregnancy & Noren L & 2002 \\
Pregnancy-related pain in the pelvis & Wu W & 2002 \\
\hline
\end{tabular}

estos podrían contribuir a la aparición del dolor lumbar gestacional y posiblemente también del posparto (7). Los cambios estudiados son esencialmente: en la postura que adopta la mujer durante el embarazo, en el contenido total de agua corporal, en el sistema endocrino y alteraciones vasculares observadas a nivel de las venas epidurales.

La postura: Existe la teoría de que cambios posturales caracterizados por un aumento de la lordosis lumbar pueden contribuir al desarrollo de dolor lumbar en este grupo de pacientes. La mayor parte del peso ganado por la embarazada está concentrado en la parte inferior de la pelvis junto con el aumento de volumen abdominal. Por esto se produciría una tendencia a inclinar el cuerpo hacia delante, lo cual la embarazada tiende a compensar inconcientemente con una posición hacia atrás de la parte superior del cuerpo por sobre la pelvis, restaurando su centro de gravedad, pero aumentando la lordosis del segmento lumbar (7). Es posible que, en mujeres susceptibles, la postura anormal en lordosis que presentan durante el embarazo, resulta en estrés de las articulaciones facetarias y ligamentos, observándose aumento de la producción de líquido sinovial con distensión de la cápsula articular lo cual produciría finalmente dolor.

Otros estudios no han podido demostrar una relación entre la magnitud de la lordosis y el dolor.

Contenido total de agua corporal: El contenido de agua corporal aumenta en forma similar tanto en la primípara como en la multípara, en un promedio de 8,5 litros (8). Esto es más que el líquido amniótico fetal y la placenta, lo cual se refleja en el aumento de la hidratación de las macromoléculas en el tejido conectivo. Esto ocurre inicialmente por el efecto aumentado natriurético de la progesterona en las primeras etapas del embarazo. Luego, el balance negativo de sodio induce la renina y la isorenina, la última producida a nivel del útero, estas a su vez activan la producción de angiotensina II con el posterior aumento de la aldosterona y la hormona antidiurética, las cuales reabsorben sodio y aumentan el contenido de agua corporal (9). Esta retención de fluido, particularmente a nivel del tejido conectivo de la columna vertebral y pelvis, aumenta la laxitud de estas articulaciones, que podría contribuir a la génesis del dolor. 
Cambios endocrinos: El efecto de la retención de fluidos recién mencionada se ve aumentado por los cambios endocrinos del embarazo, en particular por el efecto de la relaxina. La relaxina es un polipéptido de doble cadena con un peso molecular de $6500 \mathrm{KDa}$, similar a la insulina que regularía la fisiología del colágeno. Es secretada por el cuerpo lúteo y la placenta, que tendría entre sus efectos el aumento de la laxitud de los ligamentos alrededor de la pelvis y cuello uterino, a través del aumento del porcentaje de agua en estas estructuras (10). Esta laxitud, necesaria para acomodar el feto en desarrollo y facilitar el parto, podría ser causante de distensión y aumento del rango de movimiento de las articulaciones sacro ilíacas y de la sínfisis, lo cual a su vez podría causar dolor (11). Otro cambio en el embarazo, es que existiría una menor modulación del dolor por disminución de los niveles de sustancia $\mathrm{P}$ a nivel del cuerno posterior de la médula espinal, esto se ha estudiado a nivel de concentraciones salivales de este compuesto en pacientes embarazadas y no embarazadas (12). Los niveles séricos de las hormonas reproductivas y el procolágeno se han visto asociados a la aparición de dolor pélvico en el embarazo avanzado (6). Los anticonceptivos también han sido investigados, en relación a este mismo hecho, observándose hallazgos contradictorios, lo que sí se sabe es que los anticonceptivos orales influirían en el metabolismo de colágeno (6).

Congestión de vasos epidurales: Hasta el 67\% de las mujeres reportan dolor lumbar durante la noche en la segunda mitad del embarazo, esto se ha intentado explicar por la hipervolemia combinada con la obstrucción de la vena cava inferior, por el útero aumentado de tamaño, lo que podría resultar en una congestión del sistema venoso, en especial las venas extradurales dístales a la zona en conflicto de espacio, esto a su vez produciría hipoxia e irritación de las fibras amielínicas, finalmente llevando a la aparición del dolor lumbar, según postulan las teorías publicadas (13).

\section{CARACTERÍSTICAS DEL DOLOR}

La presentación clínica del dolor lumbopélvico relacionado al embarazo, varía ampliamente tanto de caso en caso, como en el tiempo en una misma paciente. Los síntomas son frecuentemente moderados, aunque también pueden ser severos e incapacitantes. Frecuentemente la aparición del dolor ocurre alrededor de la semana $18^{\underline{a}}$ de embarazo y la máxima intensidad se observaría entre la semana $24^{a}$ a $36^{a}$. El dolor durante el primer tri- mestre de embarazo puede ser un predictor fuerte de dolor en el tercero (14). Además, en el posparto el dolor desaparece en el $93 \%$ de los casos en los primeros 3 meses, el $7 \%$ de los casos restantes tienen un alto riesgo de dolor lumbar prolongado (15). La localización del dolor es frecuente en la región sacra y glútea, descrito como profundo, careciendo de distribución radicular. Además hay que tener en cuenta que la localización es muchas veces diferente a través del tiempo, migrando durante el curso del embarazo, cambiando desde lumbar a sacro (5). La naturaleza del dolor pélvico se ha descrito como tipo estocada ("stabing"), la del dolor lumbar como sordo ("dull ache") y en la zona torácica como quemante (16). En cuanto a la intensidad del dolor el promedio fluctúa entre 50 a $60 \mathrm{~mm}$ en la escala visual análoga de $100 \mathrm{~mm}$. Se ha reportado que el dolor es leve a moderado en el $50 \%$ de los casos y severo en el $25 \%$ de las embarazadas (17). El dolor posparto es de menor intensidad que el dolor presente durante el embarazo (17). Además se ha observado que el dolor pélvico es de mayor intensidad que el lumbar durante el embarazo y se invierte la situación luego del parto (18).

En las mujeres embarazadas con dolor lumbopélvico se ha observado un menor porcentaje de potenciales eléctricos en la electromiografía (EMG) de la musculatura paraespinal durante la flexión de tronco que las mujeres embarazadas sanas, las cuales a su vez tienen mejores trazados de EMG que las pacientes no embarazadas. Mens y cols (19), publicaron la hipótesis que la reducción de fuerza de las embarazadas con dolor no se encontraría relacionada con debilidad per se, sino que más bien el dolor o temor al dolor haría a la paciente más cautelosa con la fuerza realizada. Actualmente ésta hipótesis, como otras, son especulativas y deben aún someterse a estudio científico (5).

En cuanto a los cambios de la percepción de los movimientos, se ha descrito sensación de atrapamiento ("catching") de los muslos y también sensación en las piernas "como si estuvieran paralizadas", estas extrañas percepciones sugieren que algo inusual ocurre en el sistema nervioso de la paciente con dolor en la cintura pélvica relacionado al embarazo e interesantemente no se han observado en las pacientes con dolor lumbar. Por último, se han descrito cambios en la coordinación de los movimientos, observándose una cinemática distinta en mujeres con dolor lumbar posparto durante el proceso de levantar objetos, comparadas con controles sanos (20). 


\section{INCAPACIDAD ASOCIADA AL DOLOR}

Las pacientes con dolor lumbopélvico relacionado al embarazo, presentan dificultad para caminar rápido y frecuentemente están inhabilitadas para cubrir grandes distancias. Las frecuencias de problemas de incapacidad publicados en estos pacientes varían entre 21 a $81 \%$ con una media de $28 \%$ (5). Una mayor tasa de incapacidad se ha reportado para situaciones como son: levantarse desde el suelo $(97 \%)$ y dificultad o dolor al tener relaciones sexuales (82\%) (21). Varios estudios han publicado problemas durante la noche al conciliar el sueño, esto afectaría al 30\% de las embarazadas (5). Padua y cols (22), al aplicar el cuestionario de incapacidad de Roland (23), encontraron que las mujeres con dolor lumbopélvico durante el embarazo presentaban 0 puntos en el $31 \%$ de los casos, 1 a 4 puntos en el 40\%, 5-10 puntos en el $21 \%$, mas de 10 puntos en solo el $8 \%$ de las pacientes (escala de 0 a 24 puntos), concluyendo en este estudio que la mayor parte de las embarazadas no presentaban gran incapacidad asociada al dolor, siendo leve a moderada en el $92 \%$ de los casos, aunque por otra parte hay que considerar que existe un $8 \%$ de las pacientes con incapacidad severa por esta complicación. En una asociación de pacientes (24) con dolor lumbopélvico relacionado al embarazo se describieron las actividades para las cuales las embarazadas se encontraban más incapacitadas de realizar por el dolor, en orden de frecuencia descendente: los trabajos de la casa, el ejercicio, actividades con los niños, trabajo remunerado, entretenimiento, relaciones personales y sexuales.

\section{PREVALENCIA}

La prevalencia de dolor lumbopélvico durante el embarazo ha sido reportada como "muy común" y considerada como la complicación más frecuente de este, pero diferentes estudios entregan por- centajes muy variables entre si, fluctuando entre 3,9 al $89,9 \%$ (5) con un promedio de $45,3 \%$ y mediana de $49 \%$. En cuanto a la persistencia del dolor lumbar luego del parto, ha sido reportada entre 5 a $40 \%$ luego de transcurrido 6 meses después del embarazo $(25,26)$. A los 18 meses post parto este porcentaje disminuiría a 7\% (25), aunque definiéndose como residual y serio. La real prevalencia, a la cual nos enfrentamos en nuestro medio, no la podremos saber hasta definir en forma adecuada la entidad que estamos buscando, estudiar la población a la cual estamos tratando, y determinar a que factores de riesgo está sometida.

\section{FACTORES DE RIESGO}

En cuanto a los factores de riesgo (Tabla II), estos han sido estudiados ampliamente, aunque algunos aún se encuentran en discusión. Con una evidencia fuerte (con por lo menos 10 trabajos publicados a favor del factor estudiado y sin estudios en contra) figuran el trabajo estresante, dolor lumbar previo y dolor lumbopélvico relacionado al embarazo previo. La evidencia débil (con por lo menos un estudio que reporta influencia significativa y sin estudios en contra) para el peso y la estatura materna, el peso fetal, el uso de anticonceptivos orales previos, tabaquismo, anestesia epidural y labor de parto prolongado. Existiría solo evidencia conflictiva, definiéndose con estudios tanto a favor como en contra, para: edad materna, número de embarazos y etnia materna. Por último no existe evidencia sobre la densidad ósea y tampoco sobre los abortos previos como factores de riesgo en dolor lumbopélvico relacionado con el embarazo (5).

\section{TRATAMIENTO}

Las posibles intervenciones para la prevención y el manejo del dolor pélvico y lumbar relacionado

Tabla II

\section{GRADOS DE EVIDENCIA SOBRE FACTORES DE RIESGO EN POBLACIÓN CON DOLOR LUMBAR RELACIONADO AL EMBARAZO}

\begin{tabular}{llll}
\hline Evidencia fuerte & Evidencia débil & Evidencia conflictiva & Sin evidencia \\
\hline Trabajo estresante & Peso y estatura materna & Edad materna & Densidad ósea \\
Dolor lumbar previo & Peso fetal & Número de embarazos previos & Abortos previos \\
Dolor lumbopélvico relacionado & Uso de anticonceptivos & Etnia materna & \\
al embarazo previo & Tabaquismo & & \\
& Anestesia epidural & & \\
& Trabajo de parto prolongado & & \\
\hline
\end{tabular}


con el embarazo fueron motivo de la confección de una revisión sistemática por el grupo Cochrane (27), en la cual se destaca el hecho de que a pesar de ser un problema frecuente y con alto impacto en la sociedad solo existen 3 estudios relevantes (Randomized Controlled Trial o RCT) hasta el año 2001 (fecha de última modificación importante del artículo), y a través del análisis de estos se concluyó que la gimnasia acuática reduciría el dolor lumbar durante el embarazo, logrando un mayor número de mujeres que pueden seguir trabajando; el uso de almohadas especialmente diseñadas (tipo Ozzlo) ayudarían a aliviar el dolor lumbar en el último trimestre del embarazo y a mejorar el sueño; por último, este estudio concluyó, que tanto la fisioterapia como la acupuntura pueden aliviar el dolor lumbar y pélvico, y aunque se consideraron a las sesiones de acupuntura como de más ayuda que las sesiones de fisioterapia, hay que considerar que las primeras eran otorgadas en forma individual y las segundas en grupo, por lo cual podría pesar el efecto de la terapia dirigida y personalizada.

Una revisión sistemática publicada el 2003 (28) sobre el uso de terapia física para el dolor lumbar relacionado al embarazo mostró resultados similares a lo descrito en la revisión Cochrane, destacando una serie de artículos adicionales, de mala calidad metodológica, que no permiten realizar nuevas conclusiones. En un estudio reciente publicado por Kvorning y cols (29), comparó el uso de acupuntura en un grupo de pacientes embarazadas con dolor lumbar con un grupo control, observando un $60 \%$ de disminución de la sintomatología versus un $14 \%$ en el grupo control durante el período del estudio, evaluado finalmente al momento del parto, y sin observar efectos adversos de la técnica.

En cuanto a medicamentos el más comúnmente usado es el paracetamol, que ha demostrado ser inocuo para la madre y el feto en crecimiento, por períodos cortos de uso (30); en cuanto a los AINES estos no debieran usarse ya que producirían teratogénesis y mayores índices de aborto espontáneo (31). El uso de medicamentos no ha sido estudiado mediante RCT por lo cual no sabemos cuanto efecto puedan tener sobre la sintomatología de la paciente. No existen RCT que avalen el uso de cinturones trocantéricos, electroanalgesia, cese del tabaquismo y educación al paciente para la disminución del dolor (7).

El reposo en cama no tiene fundamento científico, al igual que en el dolor lumbar inespecífico, el dolor lumbar relacionado al embarazo en au- sencia de disfunción pélvica y el dolor pubiano al deambular. El reposo por más de dos días, en el caso de la embarazada de hecho prolonga la discapacidad (32). El reposo prolongado favorece el incremento de peso lo que deteriora aun más la condición de sobrecarga espinal fisiológica y acentúa la percepción de discapacidad (32).

Por otra parte, la condición del embarazo genera una discapacidad para esfuerzos mayores y posiciones mantenidas, que la ley chilena recono$\mathrm{ce}$, estas disposiciones son ampliamente ignoradas tanto por los trabajadores como las empresas, ello genera insatisfacción laboral y búsqueda de beneficios de reposo a través de la licencia médi$\mathrm{ca}$, en lugar de la modificación del régimen laboral que la propia ley señala. La legislación chilena dispone lo siguiente (33): "Durante el período de embarazo, la trabajadora que esté ocupada habitualmente en trabajos considerados por la autoridad como perjudiciales para la salud, deberá ser trasladada, sin reducción de sus remuneraciones, a otro trabajo que no sea perjudicial a su estado. Para estos efectos se entenderá, especialmente, como perjudicial para la salud todo trabajo que: a) obligue a levantar, arrastrar o empujar grandes pesos; b) exija un esfuerzo físico, incluido el hecho de permanecer de pie largo tiempo; c) se ejecute en horario nocturno; d) se realice en horas extraordinarias de trabajo, y e) la autoridad competente declare inconveniente para el estado de gravidez". Al respecto y en la medida que los empleadores y las trabajadoras no utilizan esta iniciativa legal, estas tienden a expresar el dolor como discapacidad, de tal modo de obtener a través de reposo médico la supresión de tareas de esfuerzo físico.

\section{CONCLUSIÓN}

El dolor lumbar relacionado con el embarazo es una patología de reciente estudio en la literatura médica, pese a existir una gran cantidad de artículos en las últimas dos décadas, aún no se clarifica la etiopatogenia. Desconocemos la prevalencia en nuestro medio, ya que porcentajes tan variables publicados en los artículos internacionales no son extrapolables a nuestras pacientes. En cuanto a factores de riesgo y tratamiento, aun faltan investigaciones que sean de buena calidad metodológica, aunque por el momento, a partir de los estudios ya realizados, se vislumbran algunos tratamientos que controlan la sintomatología. Por el momento, debemos conformarnos con la información disponible, y a partir de ella otorgarles 


\section{Dolor lumbar relacionado al embarazo}

\section{Anamnesis y examen físico}

Segundo y tercer trimestre
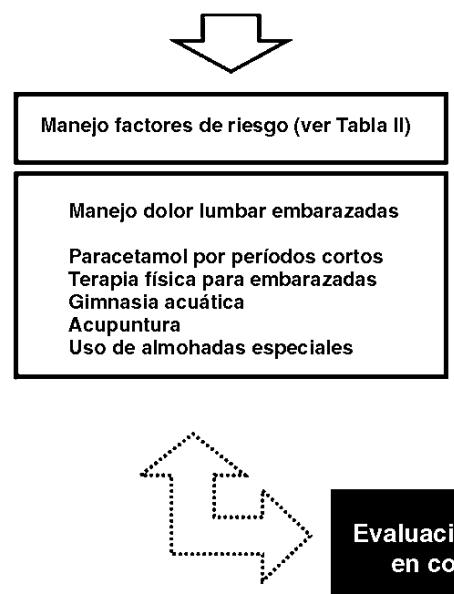

Evaluación por especialista en columna vertebral

respuestas a nuestras pacientes para una mejor calidad de vida durante la gestación (Figura 1).

\section{BIBLIOGRAFÍA}

1. Bastiaanssen J, De Bie R, Bastiaenen C, et al. A historical perspective on pregnancy-related low back and/or pelvic girdle pain. Eur J Obstet Gynecol Reprod Biol 2005;120(1):3-1.

2. Abramson D, Summer M, Wilson P. Relaxation of the pelvis joints in pregnancy. Surg Gynecol Obstet 1934;58:595-613.

3. Genell S. Studies on insufficientia pelvis (gravidarum et puerperarum). Acta Obstet Gynecol Scand 1949;28:1-33.

4. Walde J. Obstetrical and gynecological back and pelvic pains, especially those contracted during pregnancy. Obstet Gynecol Scand 1962;2:11-53.
Figura 1. Algoritmo propuesto basado en la revisión de la literatura.

\section{3 semanas}

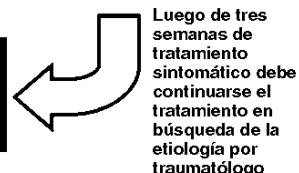

5. Wu W, Meijer O, Uegaki K, et al. Pregnancy-related pelvic girdle pain (PPP), I: Terminology, clinical presentation, and prevalence. Eur Spine J 2004; 13:575-89.

6. Mogren I, Pohjanen A. Low back pain and pelvic pain during pregnancy: prevalence and risk factors. Spine 2005;30:983-91.

7. MacEvilly M, Buggy D. Back pain and pregnancy: a review. Pain 1996;64:405-14.

8. Forsum E, Sadurskis A, Wager J. Resting metabolic rate and body composition of healthy Swedish women during pregnancy. Am J Clin Nutr 1988; 47:942-7.

9. Lapidus AM. Cambios fisiológicos maternos durante el embarazo. Pérez-Sánchez A, Donoso E (eds). En: Obstetricia, $3^{\underline{a}}$ ed. Editorial Mediterráneo 1999; capítulo 10; 174-92.

10. Szlachter N, O`Byrne EM, Goldsmith LT, et al. 
Myometrial-inhibiting activity of relaxin containing extract of human corpora lutea of pregnancy. Am J Obstet Gynecol 1980;136:584-6.

11. MacLennan A, Nicolson R, Green R, Bath M. Serum relaxin and pelvic pain of pregnancy. Lancet 1986; I:243-5.

12. Dalby P, Ramanathan S, Rudy T, et al. Plasma and saliva substance $P$ levels: The effects of acute pain in pregnant and non-pregnant women. Pain 1997; 69(3):263-7.

13. Fast A, Weiss L, Parikh S, Hertz G. Night backache in pregnancy. Hypothetical pathophysiological mechanisms. Am J Phys Med Rehabil 1989;68(5):227-9.

14. Sihvonen $\mathrm{T}$, Huttunen $\mathrm{M}$, Makkonen $\mathrm{M}$, Airaksinen O. Functional changes in back muscle activity correlate with pain intensity and prediction of low back pain during pregnancy. Arch Phys Med Rehabil 1998;79:210-2.

15. Östgaard HC, Anderson GB, Wennergren M. The impact of low back and pelvic pain in pregnancy outcome. Acta Obstet Gynecol Scand 1991;70:21-4.

16. Sturesson B, Udén G, Udén A. Pain pattern in pregnancy and "catching" of the leg in pregnant women with posterior pelvic pain. Spine 1997;22: 880-3.

17. Kristiansson P, Svärdsudd K, Von Schoultz B. Back pain during pregnancy: a prospective study. Spine 1996;21:702-9.

18. Östgaard HC, Roos-Hansson E, Zetherström G. Regression of back and posterior pelvic pain after pregnancy. Spine 1996;21:777-80.

19. Mens JM, Vleeming A, Snijders CJ, et al. Reliability and validity of hip adduction strength to measure disease severity in posterior pelvic pain since pregnancy. Spine 2002;27:674-9.

20. Commissaris DA, Nilsson-Wikmar LB, Van Dieën $\mathrm{JH}$, Hirschfeld $\mathrm{H}$. Joint coordination during wholebody lifting in women with low back pain after pregnancy. Arch Phys Med Rehabil 2002;83:1279-89.

21. Hansen A, Jensen DV, Wormslev M, et al. Symptomgiving pelvic girdle relaxation in pregnancy, II: Symptoms and clinicals signs. Acta Obstet Gynecol Scand 1999;78:111-5.
22. Padua L, Padua R, Bondi R, et al. Patient oriented assesment of back pain in pregnancy. Eur Spine $\mathrm{J}$ 2002;11:272-5.

23. Kovacs FM, Llobera $J$,Gil del Real MT, et al. Validation of the Spanish Version of the Roland-Morris Questionnaire. Spine 2002;27:538-42.

24. MacLennan AH, MacLennan SC. The Norwegian Association for Women with Pelvic Girdle Relaxation. Symptom-giving pelvic girdle relaxation of pregnancy, postnatal pelvic joint syndrome and developmental dysplasia of the hip. Acta Obstet Gyneco Scand 1997;76:760-4.

25. Albert H, Godskesen M, Westergaard J. Prognosis in four syndromes of pregnancy-related pelvic pain. Acta Obstet Gynecol Scand 2001;80:505-10.

26. Östgaard HC, Anderson GB. Postpartum low-back pain. Spine 1992;17:53-5.

27. Young G, Jewell D. Interventions for preventing and treating backage in pregnancy (Cochrane Review). In The Cochrane Library, 4, 2001. Oxford: Update Software. CD001139.

28. Stuge B, Hilde G, Vollestad N. Physical therapy for pregnancy-ralated low back and pelvic pain: a systematic review. Acta Obstet Gynecol Scand 2003; 82:983-90.

29. Kvorning N, Holmberg C, Grennert L, Aberg A, Keson J. Acupuncture relieves pelvic and low-back pain in late pregnancy. Acta Obstet Gynecol Scand 2004;83:246-50.

30. Streissguth AP, Treder RP, Barr HM, et al. Aspirin and acetaminophen use by pregnant women and subsequent child IQ and attention decrements. Teratology 1987;35(2):211-9.

31. Li D, Liu L, Odouli R. Exposure to non-steroidal antiinflammatory drugs during pregnancy and risk of miscarriage: population based cohort study BMJ 2003;327;368-74.

32. Vleeming $A$, Albert $H$, Östgaard $H$, Stuge $B$, Sturesson B. European guidelines on the diagnosis and treatment of pelvis girdle pain. WG4 Concept Version 2005.

33. Código del Trabajo, artículo 202, Ley 18620. 